\title{
The Process of Control Design for the NASA Langley Minimast Structure *
}

\author{
Gary J. Balas \\ Dept. of Aerospace Engineering and Mechanics \\ University of Minnesota \\ Minneapolis, MN 55455
}

\author{
Peter Young \\ Dept. of Electrical Engineering \\ Caltech \\ Pasadena, CA 91125
}

\author{
John C. Doyle \\ Dept. of Electrical Engineering \\ Caltech \\ Pasadena, CA 91125
}

\begin{abstract}
The NASA Langley Minimast Facility is an experimental flexible structure designed to emulate future large space structures. The Minimast system consists of a 18 bay, 20 meter-long truss beam structure which is cantilevered at its base from a rigid foundation. It is desired to use active control to attenuate the response of the structure at bay 10 and 18 due to impulse disturbances at bay 9 while minimizing actuator torque commanded from the torque wheel actuators. This paper details the design process used to select sensors for feedback and performance weights on the Minimast facility. Initially, a series of controllers are synthesized using $\mathrm{H}_{2}$ optimal control techniques for the given structural model, a variety of sensor locations and performance criteria to determine the "best" displacement sensor and/or accelerometers to be used for feedback. Upon selection of the sensors, controllers are formulated to determine the affect of using a reduced order model of the Minimast structure instead of the higher order structural analysis model for control design and the relationship between the actuator torque level and the closed-loop performance. Based on this information, controllers are designed using $\mu$ synthesis techniques and implemented on the Minimast structure. Results of the implementation of these controllers on the Minimast experimental facility are presented.
\end{abstract}

\section{Introduction}

The objective of active control on the NASA Langley Minimast Facility is to attenuate the response of the structure due to input disturbance excitations entering the structure. The disturbances enter at the midpoint of the structure via three shakers and three torque wheel actuators (TWAs) located on the tip of the structure are used for control. It is necessary to select from a number of displacement and acceleration sensors available which to employ for control feedback.

This paper describes a systematic approach to the selection of sensors to be used for feedback to achieve the performance specifications in the presences of model errors and physical constraints. Upon selection of the sensors, a group of controllers are formulated to determine the affect on performance of using a reduced order model of the Minimast structure as opposed to the higher order structural analysis model for control design. The structural model, actuators, selected sensors, uncertainty deseriptions and physical constraints are included in the control design problem formulation and controllers are formulated via $\mu$-synthesis techniques for the Minimast structure. Experimental results of these controllers are presented.

\section{The Minimast Facility}

The Minimast facility is a deployable and retractable beam truss designed to represent future deployable space trusses [1]. Figure 1, obtained from the paper by Hsieh et. al., shows the Minimast facility located at NASA Langley Research Center [2]. The Minimast facility consists of a 18 bay, 20.16 meter

\footnotetext{
"submitted to the American Control Conference in Boston, May, 1991.
}

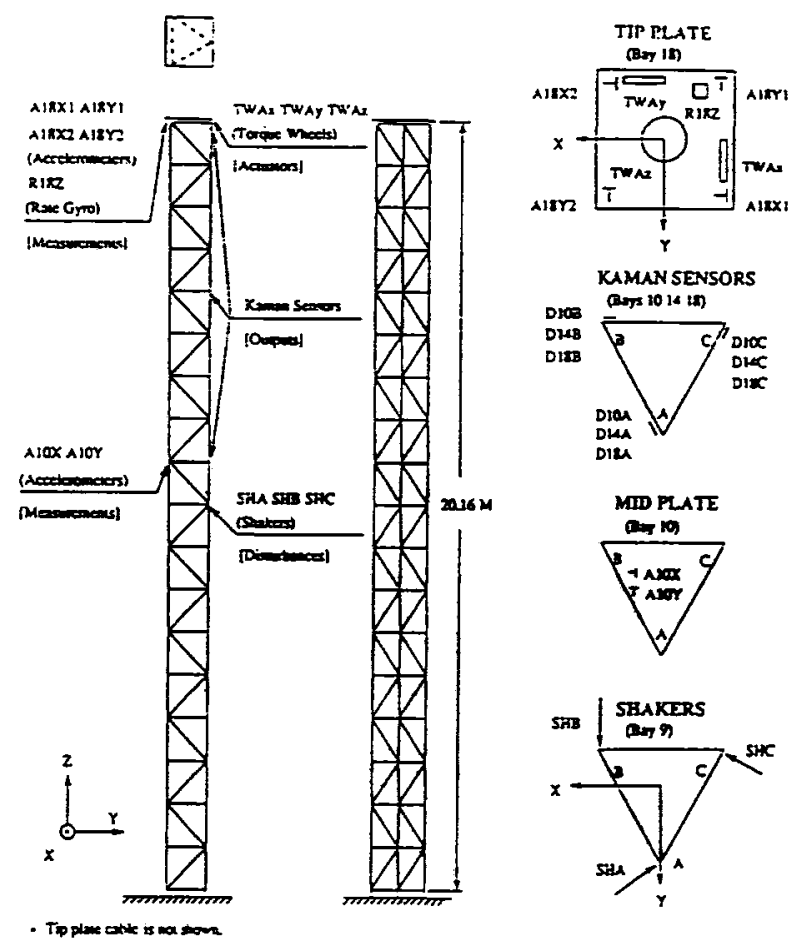

Figure 1: Minimast Facility with Actuator and Sensor Locations

long truss beam structure which is cantilever at its base from a rigid foundation. The structural members of the truss are made of a graphite/epoxy composite material with joints composed of titanium and stainless steel. A cable is attached to the tip of the Minimast structure to off load the 350lb tip mass.

\subsection{Actuators and Sensors}

The actuators and sensors to be nsed for control purposes are located on bays 10 and 18. The three torque wheel actuators used for control are located at the tip platform (bay 18) in the $x, y$ and $z$ directions. They are denoted TWAX, TWAY and TWAZ, respectively. The TWAs are represented accurately by second order transfer functions.

TWX (Input Voltage to Torque $(\mathrm{Nm}))=34861.3$ TWY (Input Voltage to Torque $(\mathrm{Nm}))=\frac{(s+23.50)(s+336.90)}{38508.9 s}$ TWZ (Is.3613+401.31) TWZ (Input Voltage to Torque $(\mathrm{Nm}))=\frac{3643.0 s}{(s+23.44)(\cdot+372.34)}$

The torque wheel actuators can saturate since the input voltage is restricted to between \pm 50 volts. This effectively limits the current to the motor with 
the back EMF limiting the speed to 62 RPM. The disturbance excitations enter the structure via three 50lb shakers attached at bay 9 of the structure. The shakers are represented as having no dynamics since their bandwidtb is far above the band vidth of the system. Placement of the actuators and shakers on the structure is shown in Figure 1.

There are two types of sensors located on bay 10 and 18 which are available for feedback: accelerometers and displacement sensors. The accelerometers, Sunstrand QA-1400, have a bandwidth of $0-200 \mathrm{~Hz}$ and very low noise characteristics. There are a totat of six accelerometers, four of them are located on the tip platform (bay 18) and two of them are located at the mid-platform (bay 10). The accelerometers are located along the $x$ and $y$ ares and are denoted A18X1, A18Y1, A18X2, A18Y2, A10X and A10Y, respectively. Only the accelerometers on bay 18 will be considered for feedback. There are three Kaman displacement sensors located at bay 10 and 18. These are mounted on a support structure parallel to the Minimast platform faces and measure displacement normal to the probe. The Kaman sensors have a range of \pm 1 inch at bay 10 and \pm 2 inches at bay 18. They are scaled to $\pm 1 \mathrm{mv} / \mathrm{mil}$ and $\pm 0.5 \mathrm{mv} / \mathrm{mil}$ at bay 10 and 18 respectively. The sensor locations can be seen in Figure 1 .

All the sensors are filtered through a third order, $10 \mathrm{~Hz}$ Bessel filter prior to digitization. The transfer function for the third order Bessel filter is:

$$
\frac{7.0653 \times 10^{5}}{s^{3}+215.734 s^{2}+1.9514 \times 10^{4} s+7.0653 \times 10^{5}}
$$

There are limitations on the excitation force, control torques and structural displacements to avoid potential damage of the Minimast facility $[1,2]$. These limitations are accounted for in the control design process.

\subsection{Structural Model}

A NASTRAN finite element model of the Minimast structure was developed and refined by NASA Langley engineers [2]. The structure is modeled as a six input, 12 output, 28 mode system. The natural frequencies, damping values and mode descriptions for the Minimast model is given in Table 1. A reduced order model of the Minimast structure is formulated based on the first five modes of the structure.

\begin{tabular}{|c|c|c|c|}
\hline Mode Number & $\begin{array}{c}\text { Predicted } \\
\text { Frequency (Hz) }\end{array}$ & $\begin{array}{l}\text { Predicted } \\
\text { Damping }\end{array}$ & $\begin{array}{c}\text { Mode } \\
\text { Description }\end{array}$ \\
\hline 1 & 0.83 & .018 & 1st Y Bending \\
\hline 2 & 0.83 & .018 & 1st $\mathrm{X}$ Bending \\
\hline 3 & 4.37 & .012 & lst Torsiona \\
\hline 4 & 6.38 & .010 & 2nd Y Bending \\
\hline 5 & 6.44 & .010 & 2nd $X$ Bending \\
\hline 6 & 14.72 & .005 & Tip Plate \\
\hline 7 & 14.83 & .005 & Diagonal \\
\hline 8 & 15.42 & .005 & 1st Axial \\
\hline 9 & 15.57 & .005 & Tip Plate \\
\hline 10 & 15.60 & .005 & Tip Plate \\
\hline 33 & 17.17 & .005 & Dizgonal \\
\hline 117 & 20.29 & .005 & Tip Plate \\
\hline 118 & 21.80 & .005 & Second Torsion \\
\hline 119 & 23.75 & .005 & Both Plates \\
\hline 120 & 25.83 & .005 & Both Plates \\
\hline 121 & 31.62 & .005 & 3rd Y Bending \\
\hline 122 & 33.10 & .005 & 3rd $X$ Bending \\
\hline 123 & 39.14 & .005 & 3rd Torsion \\
\hline 126 & 42.46 & .005 & 4th Y Bending \\
\hline 127 & 45.95 & .005 & 4th X Bending \\
\hline 128 & 54.14 & .005 & Tip Plate \\
\hline 129 & 57.25 & .005 & 4th Torsion \\
\hline 132 & 60.85 & .005 & Mid Plate \\
\hline 133 & 62.68 & .005 & Tip Plate \\
\hline 134 & 66.04 & .005 & Tip Plate \\
\hline 135 & 72.18 & .005 & 5th Y Bending \\
\hline 136 & 72.37 & .005 & 5th X Bending \\
\hline 137 & $7 \overline{3.34}$ & .005 & 5th Torsion \\
\hline
\end{tabular}

Table 1: Natural Frequencies, Damping Levels and Modes of the Minimast Structure

\section{Control Objective}

The objective of active control is to attenuate the structural response of the Minimast structure due to external disturbances entering through the exciters at bay 9 . The control actuators are torque wheels which are located on the tip platform of the Minimast structure. A number of sensors are available to the engineer and their selection for use in feedback is left to the discretion of the individual investigator. It is desired to use only accelerometers if they are able to provide sufficient information to the controller. Accelerometers are preferred for space applications due to their long history of space flight, their ease of placement on the atructure and stand alone capabilities. It is desired to avoid the use of displacement sensors since they require a reference point or support structure for measurement.

\section{Problem Formulation}

A series of controllers are designed for three different performance objectives and five different feedback sensor configurations in an effort to select the sensors pairs to be used for feedback. The three performance objectives are to attenuate the structural response of Minimast at:

- Bay 10 and 18 displacement sensors

- Bay 18 displacement sensors

- Bay 10 displacement sensors

subject to external disturbances entering at bay 9 .

The five feedback sensor configurations to be evaluated are:

- Bay 18 accelerometers only

- Bay 18 accelerometers and Bay 18 displacement sensors

- Bay 18 accelerometers, Bay 10 and 18 displacement sensors

- Bay 10 and 18 displacement sensors

- Bay 18 displacement sensors

The following questions are to be determined:

1. Can accelerometers alone be used as feedback sensors to achieve the performance objective?

2. Is enough information measured such that the problem approaches a state feedback control design problem?

3. Are the same characteristics evident on the full order and reduced order control design models?

The interconnection structure describing the control problem formulation is shown in figure 2. The input disturbances are modeled as 0.1 second, $50 \mathrm{~N}$ impulses into the exciters. This set of disturbances is represented in the problem formulation by the filter, Dist_wt, whose transfer function is $\frac{155}{+31}$. The sensor noise for the Sunstrand accelerometers and Kaman displacement sensors are very small and taken to be $2 \times 10^{-5}$ for nois18, nois 10, and noisac. The controllers will be implemented via a digital computer at a sample rate of $80 \mathrm{~Hz}$. The sample time delay is accounted for via a Pade delay, Pade_Delay, whose transfer function representation is $\frac{1-.01255}{1+0.035}$. The filter block in the figure represents the third order, $10 \mathrm{~Hz}$ Bessel filters inline with all the sensors prior to being input to the controller $\mathrm{K}$. The torque actuators are included in the problem formulation via the block Torq-act. Transfer function representations of the filters and torque actuators are provided in the Actrators and Sensors section. The Minimast structure model, Minimast, is the six input, ten output, 28 mode model. Comparisons of results between employing the five mode and 28 mode model will be discussed.

The torque level restrictions on the torque wheel actuators are included into the problem formulation via the Act_mag block. The actuator magnitude weight is selected to correspond with the size of the input disturbances. For impulse disturbances of size $50 \mathrm{~N}$ entering the shakers, the torque wheel actuators will be restricted to a maximum input of \pm 1 volt with the Act_mog 


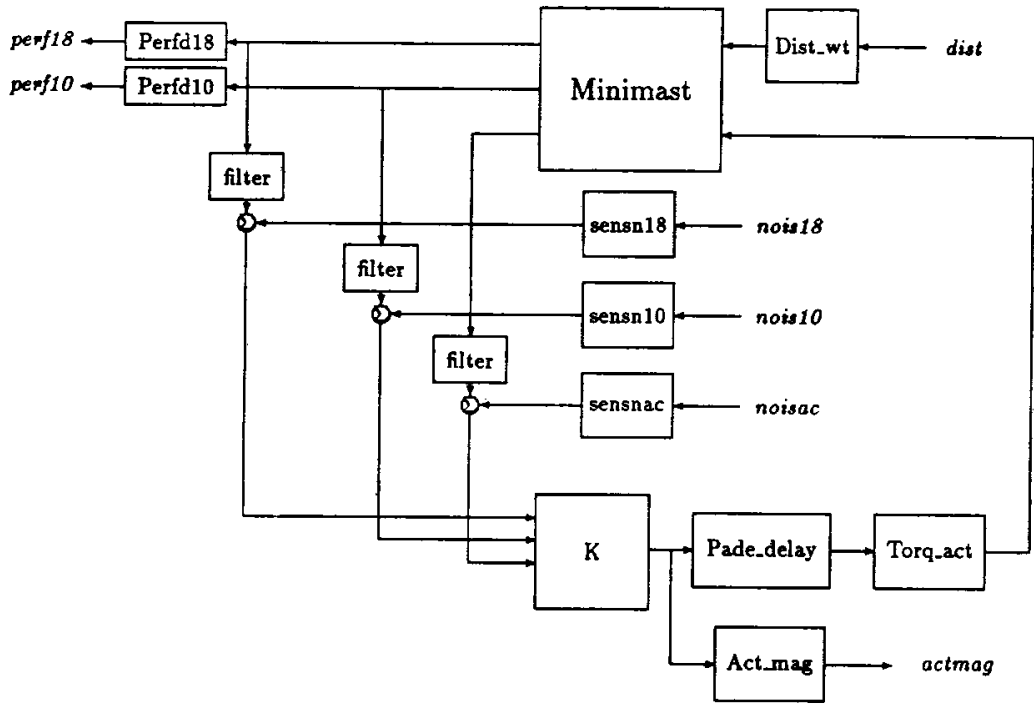

Figure 2: Interconnection Structure for Sensor Selection

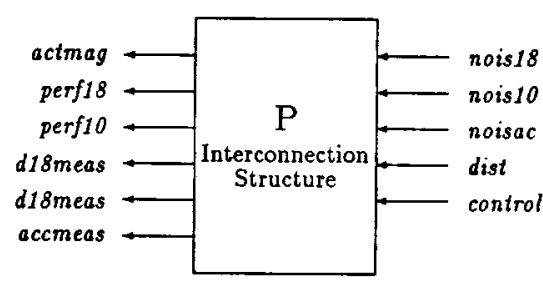

Figure 3: Control Design Plant

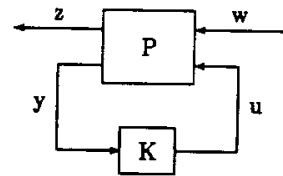

Figure 4: Linear Fractional Interconnection scaling set to 1 . This corresponds to $\pm 80 \mathrm{Nm}$ of torque. The performance objective of attenuating the vibration of the structure is included via weights on the displacement sensors at bay 10 and 18. The scalings are chosen to have the $H_{\infty}$ norm of the closed-loop transfer functions between the disturbance inputs dist and the displacement outputs be smaller than 1 . This leads to selection of Perfd18 to be 1500 and Perfd 10 to be 3000 . Measuring performance in terms of damping levels, the performance weights would require the closed-loop system to have approximately $50 \%$ damping of the first three modes. The block diagram of the sensor selection problem is shown in Figure 2 and the interconnection structure used for control design is shown in Figure 3.

\section{Control Designs}

\subsection{Sensor Selection}

Controllers are designed for the problem formulation using $\mathrm{H}_{2}$ optimal control design techniques $[3,4,5]$. The optimal $H_{2}$ controller will minimize the twonorm $\left(\|\cdot\|_{2}\right)$ of the closed-loop transfer function in Figure 4 from $w$ to $z$ which is denoted $T_{z w}$

The optimal $\mathrm{H}_{2}$ controller has the well known separation structure such that the squared two-norm of the closed-loop transfer function $T_{z w}$ is equal to the squared two-norm of the full information problem (FI) plus the squared two-norm of the output estimation problem (OE). This is also equal to the squared two-norm of the disturbance feedforward problem (DF) plus the squared two-norm of the full control problem (FC). That is

$$
\left\|T_{x w}\right\|_{2}^{2}=F I^{2}+D F^{2}=O E^{2}+F C^{2}
$$

The full information problem is better known as the state feedback problem. The selection of feedback sensors problem is dominated by the state feedback problem.

For each performance objective and set of feedback sensors the corresponding outputs of the interconnection structure are selected and a control design is synthesized. The selection criteria is based on the corresponding $\mathrm{H}_{2}$ norm of the full information, output estimation, disturbance feedforward, and full control cases. In addition to these criteria, the weighted infinity norm $\|$. $\|_{\infty}$ from disturbance to performance and from disturbance to control signal magnitude is evaluated. No uncertainty models are included in problem description for the selection of the sensors. The nomenclature used in table is:

$$
\begin{aligned}
& \text { FI - Full Information } H_{2} \text { norm } \\
& \text { OE - Output Estimation } H_{2} \text { norm } \\
& \text { DF - Disturbance Feedforward } H_{2} \text { norm }
\end{aligned}
$$
FC - Full Control $\mathrm{H}_{2}$ norm
CM - $\|\cdot\|_{\infty}$ of disturbance to control magnitude
DP - $\|\cdot\|_{\infty}$ of disturbance to displacements

The results in Table 2 indicate that use of accelerometers only for feedback with performance specifications on bay 10 and 18 incorporates all the characteristics of interest in this control design problem. Bay 18 accelerometers lead to almost a full information (state feedback) control design problem with the full information part of the control design problem dominates the output estimation problem. This implies that one is able to easily observe all the information required for control design and the difficult part is controlling the system via the torque wheel actuators.

Measuring accelerations at bay 18 is better for performance than measuring displacements at bay 18 or 10 and is as good as measuring bay 10 and 18 displacements. These results also indicate that little is gained from feeding back the displacement sensors and bay 10 and 18 in addition to the bay 18 accelerometers. All results in Table 2 are all based on the full order Minimast model. Results from using the reduced order model, shown in Table 3, correspond directly with the results obtained with the full order model. Hence only bay 18 accelerometers will be fedback for control purposes and the reduced order model will be used in the control problem formulation. The truncated struetural modes of the full order model are accounted for in the final control design via an additive uncertainty description.

\subsection{Actuator Magnitude Level Weight}

In an effort to determine the affect of the actuator torque level on the system performance, the actuator magnitude weight Act_mag is varied in the control designs. The actuator magnitude weight is scaled from 8 to 0.1 which corresponds to actuator signal levels varying from $\pm 10 \mathrm{~N} / \mathrm{m}$ to $\pm 800 \mathrm{~N} / \mathrm{m}$. It is of interested to determine the importance of the actuator magnitude scaling on the performance of the control designs and the appropriate level of magnitude weight for the system. The control design problem is the same as shown in Figure 2 but the actuator magnitude weight Act_mag is varied. The Minimast model is the full order system, performance is associated with attenuating the disturbances at bay 10 and 18 and the accelerometers at bay 18 are used for feedback.

Time history are generated with an input 0.1 second, $50 \mathrm{~N}$ unit step into shaker A. The open-loop tip displacement response at bay 18 is attenuated to $2 \%$ of its initial excitation within 50 seconds and bay 10 the response is attenuated within 40 seconds. Two control designs are presented. The controller designed with Act_mag set to 1 attenuates the response at bay 18 within 1.5 seconds and at bay 10 within 1.7 seconds. The time simulations of the open-loop system and the two closed-loop systems are shown in Figure 5. The controller design with Act_mag set to 1 generates $57 \mathrm{Nm}, 102 \mathrm{Nm}$ and $28 \mathrm{Nm}$ of torque from torque actuators $\mathrm{X}, \mathrm{Y}$ and $\mathrm{Z}$ respectively. Changing the 
Act_mag to 8 leads to the displacement at bay 18 being attenuated within 26 seconds and at bay 10 within 20 seconds. The maximum torque requirements are $7 N \mathrm{~m}, 12 \mathrm{Nm}$ and $8 \mathrm{Nm}$ from torque actuators $\mathrm{X}, \mathrm{Y}$, and $\mathrm{Z}$ respectively. The commands to the TWAY for each controller are shown in Figure 6 The trend is the same for other Act_mag weightings. Hence severly limiting the torque command signals will drastically reduce the closed-loop vibration attenuation properties of the controller. This same trend is noted when implementing controllers on the Minimast experimental facility.

\subsection{Actuator Frequency Shaped Magnitude Weights}

It is of interest to determine the effect of limiting high frequency actuator command signals has on the performance of the control designs. To investigate this issue, the magnitude weight of the actuator signal is scaled with a frequency dependent filter. This acts as a high pass filter to limit high frequency control signals. The interconnection structure in Figure 2 is used but now the Act_mag weight has dynamics. Three different actuator weights are used:

$$
\begin{aligned}
& \text { Act_mag1 }=100 \frac{+20}{3+3000} \\
& \text { Act_magz }=100 \frac{+5+5}{++500} \\
& \text { Act_mags }=\left(90 \frac{+20}{5+2000}\right)^{2}
\end{aligned}
$$

The addition of a high frequency filter to the actuator magnitude weights leads to the same level of low frequency performance as before but the high frequency structural modes are not as highly damped as in previous control designs. In the control design using Actmag1, there was little change in the performance and torque levels in the simulation. The second actuator magnitude weight, Act_mag2, more severly limited the low frequency actuator response. This lead to a slower time response of the system with little or no additional damping of the second bending modes and high natural frequencies. The damping level of the second structural mode differs slightly from the open-loop model.

To reflect the desire for good low frequeney response while restricting the amount of high frequency attenuation, the third frequency shaped actuator magnitude weight Act_mags is formulated. The closed-loop response of the controller syntheaized with this weight had similar properties to the controller synthesized with Act_mag1 although the second bending mode was not attenuated as much. These same trends are also found using the reduced order model. Therefore the results indicate that in order to achieve low frequency performance of the structure, significant actuator signal levels are required and that the large actuator commands are not due to the high frequency modes of the Minimast facility.

\section{Experimental Control Design}

Results from the fedback sensor selection and actuator magnitude weight control problems along with uncertainty models of the Minimast facility are incorporated into the experimental control problem formulation. Figure 2 forms the basis of the control problem interconnection structure with additional uncertainty weights included to account for modeling errors. Controllers are designed for the modified interconnection strueture using $\mu$-synthesis techniques and implemented on the Minimast facility $[5,6,7,8]$.

Input multiplicative uncertainty is included in the block diagram between the torque wheel actuators model and the input to the Minimast model. The input uncertainty weight is selected to be a constant $2 \%$ acrose frequency reflecting a significant amount of confidence in the TWAs models. Unmodeled high frequency modes of the Minimast structure are accounted for in the problem formulation via an additive uncertainty weight wrapping around the plant. The transfer function of the additive weight is

$$
22.222\left(\frac{s^{2}+25.46 s+324}{s^{2}+1414 s+10^{6}}\right)\left(\frac{s^{2}+42.43 s+900}{s^{2}+282.84 s+40000}\right)
$$

This weight includes sufficient low frequency uncertainty, in addition to its high frequency components, to prevent the controller from trying to attenuate rigid body modes of the support structure. The bay 18 performance weight Perf18 is selected as 4000 and the bay 10 performance weight Perf10 is taken as $20000 \frac{1+5}{1+50}$. The actuator magnitude weight Act_mag is 0.4 . The control design achieved robust performance for the given uncertainty models and performance specifications. A more detailed description of the controllers
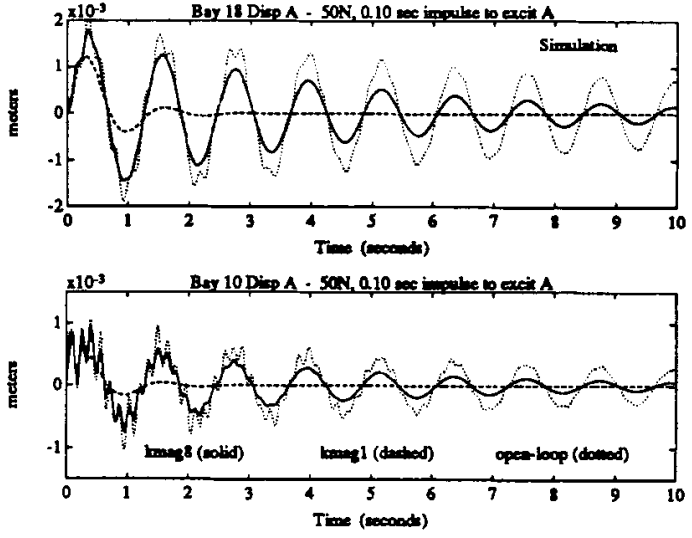

Figure 5: Time History Simulations (Displacements)

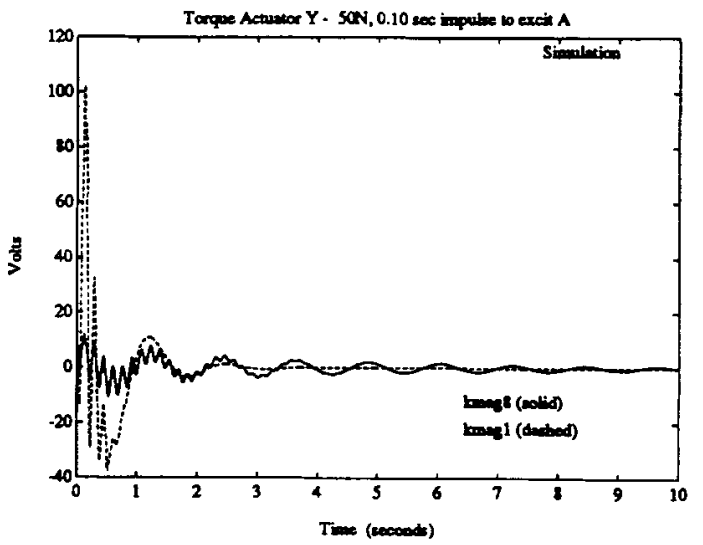

Figure 6: Time History Simulations (Torque Commands)
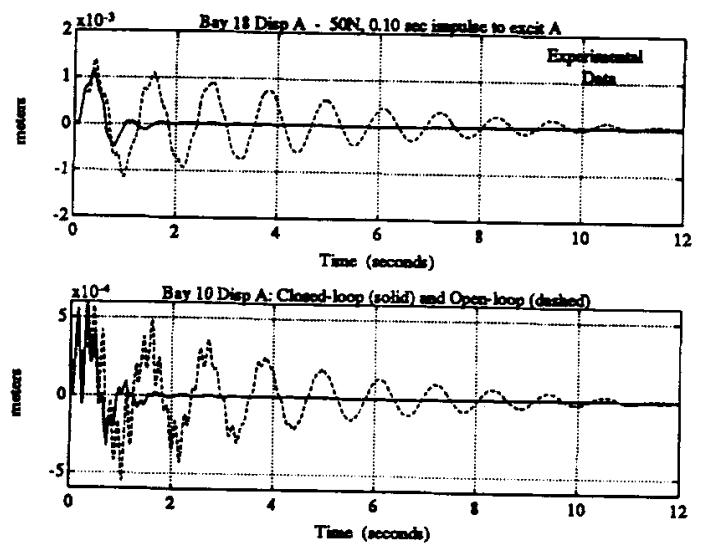

Figure 7: Experimental Displacement Time Response

designed via $\mu$-synthesis for the Minimast facility is presented in the Caltech CSI/GI final report [9].

For implementation, the controller is discretized at $80 \mathrm{~Hz}$. The experimental open-loop response of the Minimast structure at Bay 10 and 18 to a 0.1 second, $50 \mathrm{~N}$ impulse disturbance to exciter $\mathrm{A}$ is shown in Figure 7. Only the $\mathrm{X}$ displacement sensor is shown for Bay 10 and 18 since the other sensor responses are similar. One notices that there is approximately three times higher damping in the first modes of the physical structure than in the structural model. Figure 7 also shows the closed-loop displacements at bay 10 and 18 to the input disturbance with the $\mu$-synthesis controller implemented. Figure 8 is the time response of input voltage commands to the TWAs during the excitation. The torques generated by the TWAs can be determined via the TWA models which are shown in Figure 8. 


\section{Summary}

$\mathrm{H}_{2}$ optimal control techniques are used to select a set of feedback sensors for control design. The results indicate that using only bay 18 accelerometers on the Minimast structure for feedback with performance specifications on bay 10 and 18, all the characteristics of interest are incorporated in the control design problem. It is found that measuring accelerations at bay 18 is better for performance than measuring displacements at bay 10 or 18 and is as good as measuring bay 10 and 18 displacements. These same results are observed on the full and reduced order design models. The control design problem on the Minimast facility is a direct tradeoff between the actuator magnitude and the amount of closed-loop vibration attenuation achieved.

These results are combined with descriptions of modeling errors to synthesize controllers via $\mu$-synthesis techniques. Experimental results of the $\mu$-synthesis controller indicate that excellent vibration attenuation levels are achieved with bay 18 accelerometers alone used for feedback.

\section{Acknowledgements}

The authors would like to thank: Kyong Lim, Jeff Sullam Suresh Joshi, Sherri Tanner, Rudeen Smith-Taylor and all the Minimast people at NASA Langley Research Center for their assistance. This work was supported under the NASA Control/Structure Interaction (CSI) Guest Investigation program headed by Jerry Newsome.

\section{References}

1. R. Pappa, J. Sulla et. al., "Minimast CSI Testbed - User's Guide," NASA Langley Research Center, Hampton, VA, March, 1989.

2. C. Hsieh, J.H. Kim, and R.E. Skelton, "Closed-loop lab tests of NASA's Mini-Mast," Proceedings of the American Control Conference, May, 1990, pp. 1435-1440.

3. J.C. Doyle, K. Glover, P.P. Khargonekar, and B.A. Francis, "Statespace solutions to standard $\mathrm{H}_{2}$ and $H_{\infty}$ control problems," IEEE Trans. Auto. Control, vol. AC-34, no. 8, August 1989, pp 831-847.

4. K. Glover, and J. Doyle (1988). "State-space formulae for all stabilizing controllers that satisfy an $H_{\infty}$ norm bound and relations to risk sensitivity," Systems and Control Letiers, vol. 11, pp. 167-172.
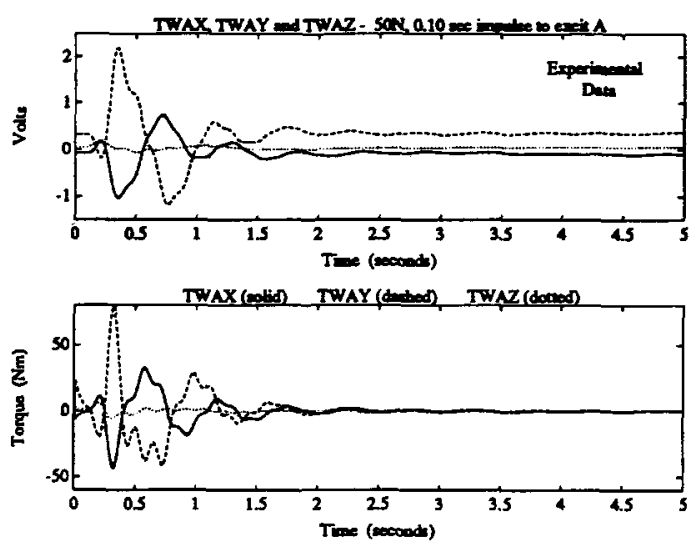

Figure 8: Experimental Torque Wheel Actuator Commands

5. Balas, Doyle, Glover, Packard and Smith, " $\mu$-Analysis and synthesis toolbox: $\mu$-Tools," MUSYN Inc. and The Mathworks Inc., December, 1990.

6. G.J. Balas, "Robust control of flexible structures: theory and experiments," Ph.D. Thesis, California Institute of Technology, Pasadena, 1989.

7. A.K. Packard, "What's new with $\mu$ : structured uncertainty in multivariable control," Ph.D. Thesis, University of California at Berkeley, 1988.

8. G.J. Balas and J.C. Doyle, "Robustness and performance tradeofts in control design for flexible structures," $29^{\text {th }}$ IEEE CDC, Honolulu, Hawaii, December, 1990.

9. G.J. Balas, J.C. Doyle and P. Young, "CSI/GI Final Report: Control design on the NASA Langley Minimast Facility," in preparation, March, 1991.

\begin{tabular}{|c|c|c|c|c|c|}
\hline \multirow[b]{2}{*}{$\begin{array}{l}\text { Perf. } \\
\text { Specs }\end{array}$} & \multicolumn{5}{|c|}{ Feedback Sensors } \\
\hline & $\begin{array}{c}\text { Accelerometers } \\
\text { Bay } 18\end{array}$ & $\begin{array}{c}\text { Accelerometers } \\
\text { Bay } 18 \\
\text { Displacement } \\
\text { Sensors } \\
\text { Bay } 10 \text { and } 18\end{array}$ & $\begin{array}{c}\text { Accelerometers } \\
\text { Bay } 18 \\
\text { Displacement } \\
\text { Sensors } \\
\text { Bay } 18 \\
\end{array}$ & $\begin{array}{l}\text { Displacement } \\
\text { Sensors } \\
\text { Bay } 10 \text { and } 18\end{array}$ & $\begin{array}{c}\text { Displacement } \\
\text { Sensors } \\
\text { Bay } 18 \\
\end{array}$ \\
\hline \multirow{6}{*}{$\begin{array}{l}\text { Bay } 18 \\
\text { and } \\
\text { Bay } 10\end{array}$} & $F I=3.641$ & $\mathrm{FI}=3.641$ & $F I=3.641$ & $F I=3.641$ & $\overline{F I}=3.641$ \\
\hline & & $O E=0.300$ & $O E=0.337$ & $O E=0.476$ & $O E=2.152$ \\
\hline & & $D F=3.648$ & $\mathrm{DF}=3.648$ & $\mathrm{DF}=\mathbf{3 . 6 5 5}$ & $\mathrm{DF}=\mathbf{3 . 8 1 3}$ \\
\hline & $\mathrm{FC}=0.754$ & $\mathrm{FC}=0.189$ & $\mathrm{FC}=0.254$ & $\mathrm{FC}=0.351$ & $F C=1.830$ \\
\hline & $\mathrm{CM}=1.340$ & $\mathrm{CM}=1.333$ & $\mathrm{CM}=1.336$ & $C M=1.337$ & $\mathrm{CM}=1.277$ \\
\hline & & $\mathrm{DP}=1.487$ & $\mathrm{DP}=1.485$ & $\mathrm{DP}=1.485$ & $\mathrm{DP}=1.646$ \\
\hline \multirow{6}{*}{ Bay 10} & & $\mathrm{FI}=2.942$ & $\mathrm{FI}=2.942$ & $\mathrm{FI}=2.942$ & $\overline{F I}=2.942$ \\
\hline & & $O E=0.380$ & $O E=0.430$ & $O E=0.525$ & $O E=1.887$ \\
\hline & $=2.943$ & $D F=2.961$ & $\mathrm{DF}=2.963$ & $\mathrm{DF}=2.929$ & $\mathrm{DF}=\mathbf{3 . 0 0 9}$ \\
\hline & $\mathrm{FC}=0.554$ & $\mathrm{FC}=0.189$ & $\mathrm{FC}=0.247$ & $\mathrm{FC}=0.341$ & $\mathrm{FC}=1.779$ \\
\hline & & $\mathrm{CM}=1.260$ & $\mathrm{CM}=1$ & $\mathrm{CM}=1.263$ & $\mathrm{CM}=1.215$ \\
\hline & $\mathrm{DP}=1.447$ & $\mathrm{DP}=1.429$ & $D P=1.425$ & $D P=1.424$ & $D P=1.908$ \\
\hline \multirow{6}{*}{ Bay 18} & & $\mathrm{FI}=2.966$ & $\mathrm{FI}=2.966$ & $\overline{F I}=2.966$ & $\mathrm{FI}=2.966$ \\
\hline & $\mathrm{OE}=0.498$ & $\mathrm{OE}=0.170$ & $\mathrm{OE}=0.200$ & $\mathrm{OE}=0.265$ & $O E=0.827$ \\
\hline & $\mathrm{DF}=2.963$ & $\mathrm{DF}=2.970$ & $D F=2.972$ & $\mathrm{DF}=2.976$ & $\mathrm{DF}=\mathbf{3 . 0 4 9}$ \\
\hline & $\mathrm{FC}=0.512$ & $\mathrm{FC}=0.016$ & $\mathrm{FC}=0.059$ & $\mathrm{FC}=0.085$ & $\mathrm{FC}=0.428$ \\
\hline & $C M=1.340$ & $\mathrm{CM}=1.279$ & $C M=1$ & $\mathrm{CM}=1.278$ & $\mathrm{CM}=1.251$ \\
\hline & $\mathrm{DP}=1.157$ & $\mathrm{DP}=1.381$ & $\mathrm{DP}=1.383$ & $\mathrm{DP}=1.383$ & $\mathrm{DP}=1.455$ \\
\hline
\end{tabular}

Table 2: Full Order Model Sensor Selection 


\begin{tabular}{|c||c|c|c|c|c|}
\hline \multicolumn{1}{||c||}{} & \multicolumn{5}{c|}{ Feedback Sensors } \\
Perf. & Accelerometers & $\begin{array}{c}\text { Accelerometers } \\
\text { Bay 18 } \\
\text { Displacement } \\
\text { Sensors }\end{array}$ & $\begin{array}{c}\text { Accelerometers } \\
\text { Bay 18 } \\
\text { Displacement } \\
\text { Sensors }\end{array}$ & $\begin{array}{c}\text { Displacement } \\
\text { Sensors }\end{array}$ & $\begin{array}{c}\text { Displacement } \\
\text { Sensors } \\
\text { Specs }\end{array}$ \\
& Bay 18 & Bay 10 and 18 & Bay 18 & Bay 10 and 18 & Bay 18 \\
\hline & $\mathrm{FI}=3.621$ & $\mathrm{FI}=3.621$ & $\mathrm{FI}=3.621$ & $\mathrm{FI}=3.621$ & $\mathrm{FI}=3.621$ \\
Bay 18 & $\mathrm{OE}=0.654$ & $\mathrm{OE}=0.275$ & $\mathrm{OE}=0.298$ & $\mathrm{OE}=0.560$ & $\mathrm{OE}=2.087$ \\
and & $\mathrm{DF}=3.607$ & $\mathrm{DF}=3.629$ & $\mathrm{DF}=3.630$ & $\mathrm{DF}=3.653$ & $\mathrm{DF}=3.801$ \\
Bay 10 & $\mathrm{FC}=0.728$ & $\mathrm{FC}=0.130$ & $\mathrm{FC}=0.165$ & $\mathrm{FC}=\mathbf{0 . 2 9 2}$ & $\mathrm{FC}=1.738$ \\
& $\mathrm{CM}=1.336$ & $\mathrm{CM}=1.330$ & $\mathrm{CM}=1.332$ & $\mathrm{CM}=1.335$ & $\mathrm{CM}=1.277$ \\
& $\mathrm{DP}=1.526$ & $\mathrm{DP}=1.487$ & $\mathrm{DP}=1.486$ & $\mathrm{DP}=1.484$ & $\mathrm{DP}=1.641$ \\
\hline
\end{tabular}

Table 3: Reduced Order Model Sensor Selection 